\title{
BARRERAS LABORALES PARA LA INCLUSIÓN DE LA POBLACIÓN CON DISCAPACIDAD EN LA LOCALIDAD DE CIUDAD BOLÍVAR.
}

\author{
Employment barrier's to the disabled people
}

\author{
Camila Andrea Gamboa Cárdenas \\ cagamboa@unicolmayor.edu.co \\ Chanel Juliana Cortés Huertas \\ Cjcortes@unicolmayor.edu.co \\ Universidad Colegio Mayor de Cundinamarca \\ Tecnología en Asistencia Gerencial \\ Colombia
}

Resumen

La población en condición de discapacidad de la localidad Ciudad Bolívar, está conformada por 8.164 personas, la cuales cuenta con capacidad de trabajar según el Departamento de Nacional de Estadísticas DANE, (2010); del total de esta población solo el 21\%, desarrolla alguna productiva, a pesar de los esfuerzos gubernamentales (Martínez, A. M., Uribe, A. F., y Velázquez, H. J.,2015), se desconoce desde el contexto de los programas distritales de la alcaldía, las causas que afectan la inclusión laboral con el fin de formular estrategias que permitan su acceso al mundo productivo. La investigación se realiza haciendo análisis de fuentes secundarias que permitan determinar el alcance y la forma de acercamiento a la población desde el análisis de experiencias exitosas en otros contextos y el trabajo de campo y la respectiva consulta a la población con condición de discapacidad, beneficiaria de programas distritales en la localidad. Los resultados del estudio orientan a los empleadores sobre el potencial y la necesidad de inclusión en la localidad y la articulación con los esfuerzos locales.

Palabras clave: barreras laborales, Ciudad Bolívar, discapacidad, inclusión y programas distritales 


\section{INTRODUCCIÓN}

Las personas en condición de discapacidad enfrentan retos a nivel mental, físico e intelectual. Desde la investigación, se han abordado soluciones biomédicas y sociales; en particular estos desafíos sociales implican la vinculación laboral para hacerles parte activa y productiva de las sociedades y responder a los desafíos de diversidad, disminución de la población como parte de la fuerza laboral y la actividad productiva como una solución a riesgos psicosociales. Para Smith (2009), la discapacidad se define como una condición fija de enfermedades preestablecidas que no se pueden cambiar, sin embargo, experiencias exitosas nos demuestran que pueden sumar desde la inclusión social y económica.

En la Localidad de Ciudad Bolívar según estadísticas del DANE(2010), se encuentra localizada alrededor de 8164 personas entre los 18 y los 60 años que presentan una discapacidad, rango de edad el cual es manejado por la población a la cual tenemos acceso, que incluye la mayoría de edad en Colombia para poder acceder al mundo laboral, en relación a esta estadística el DANE arroja que solo 1772 personas en condición de discapacidad se dedican a actividades productivas y solo 350 están en búsqueda de un empleo, es decir solo el 4\% de la población registra la búsqueda, sin embargo desde el contexto de la fundación se ha identificado el interés por acceder a alguna actividad productiva que les permita el mejoramiento de la calidad de vida propia y de sus familias (E. Norman-Acevedo, 2018).

En el desarrollo de la investigación, se tienen en cuenta herramientas y aportes realizados en otras investigaciones que presentaron proyectos en torno a la vinculación de jóvenes víctimas del conflicto armado en Colombia al mundo laboral en la localidad de Bosa como manera de identificar las oportunidades y dificultades laborales que se presentan a jóvenes de bajos recursos para vincularse a una institución de manera laboral (De la Cruz,J.F., 2015), caso de la Universidad del Rosario y proyectos relacionados con procesos de inclusión como el proyecto 1113 en la Localidad de Ciudad Bolívar, que tiene que ver con la inclusión no solo al mundo laboral, también en temas de educación y de salud de la Universidad Uniminuto (Farfán, M. H., y Virgüez, O., 2018), en donde se explica la importancia, los fundamentos y las posibles áreas de intervención para la inclusión y la vinculación laboral de adolescentes y jóvenes (Navarrete, Padrón y Silva, 2013) de localidades con estratos entre 1 y 2 sin dejar en condición de discapacidad, además de hacer un estado del arte de experiencias en otros países.

Método

La investigación es de tipo exploratorio mixta ya que busca saber qué tan amplio es el acceso al mundo laboral para una persona en condición de discapacidad y obtener profundo conocimiento a través de 
los datos arrojados, para su caracterización y análisis de la población desde los motivos y causas de bajo acceso al mercado laboral. La investigación será descriptiva transversal (Hernández Sampieri, R., Fernández Collado, C., y Baptista Lucio, P.,2010; Daza-Orozco, 2019), mediante una encuesta, realizada a los usuarios de los programas en el marco del convenio 1113 denominado "por una ciudad incluyente y sin barreras" en condición de discapacidad de la localidad Ciudad Bolívar en Bogotá.

\begin{tabular}{|c|c|c|c|}
\hline \multicolumn{4}{|c|}{$\begin{array}{l}\text { Propósito General: Analizar los desafíos que enfrentan las personas con discapacidad y su vida laboral de la localidad } \\
\text { Ciudad Bolívar. }\end{array}$} \\
\hline Objetivos Específicos & Variables & Subcategoría & Indicadores \\
\hline \multirow[b]{2}{*}{$\begin{array}{l}\text {-Caracterizar la población en } \\
\text { condición de discapacidad de } \\
\text { la Localidad de Ciudad } \\
\text { Bolívar que han accedió o } \\
\text { intentado acceder al mundo } \\
\text { laboral. }\end{array}$} & \multirow{3}{*}{$\begin{array}{l}\text { Capital humano, } \\
\text { Discapacidad }\end{array}$} & Remuneración, Equidad & $\begin{array}{l}\text { Definición } \\
\text { Bienestar, } \\
\text { Crecimiento }\end{array}$ \\
\hline & & \multirow[t]{2}{*}{$\begin{array}{l}\text { Calidad de las ocupaciones } \\
\text { que desempeñan los sujetos }\end{array}$} & $\begin{array}{l}\text { Definición } \\
\text { Tipo de estudio } \\
\text { Genero del sujeto } \\
\text { Edad de los sujetos } \\
\text { Incentivos } \\
\text { Motivaciones } \\
\text { Condiciones de Igualdad }\end{array}$ \\
\hline $\begin{array}{l}\text {-Identificar las dificultades } \\
\text { que se presentan a una } \\
\text { persona en condición de } \\
\text { discapacidad para acceder a } \\
\text { un vínculo laboral. }\end{array}$ & & & $\begin{array}{l}\text { Definición } \\
\text { Autorrealización, Sentirse } \\
\text { competente y útil } \\
\text { Autoestima, Organización } \\
\text { Liderazgo }\end{array}$ \\
\hline $\begin{array}{l}\text {-Contrastar la caracterización } \\
\text { de la población en relación } \\
\text { con las dificultades } \\
\text { presentadas para la } \\
\text { vinculación al mundo laboral }\end{array}$ & $\begin{array}{l}\text { Integración, trabajo } \\
\quad \text { sostenible }\end{array}$ & $\begin{array}{l}\text { Motivación Laboral, Buen } \\
\text { trabajo, inclusión }\end{array}$ & $\begin{array}{l}\text { Productividad } \\
\text { Reconocimiento } \\
\text { Relaciones Interpersonales en } \\
\text { el Trabajo } \\
\text { Rentabilidad } \\
\text { Satisfacción Laboral } \\
\text { Seguridad Laboral }\end{array}$ \\
\hline
\end{tabular}

Tabla 1. Relación con los objetivos y las variables consultadas a población en discapacidad de la Resultados localidad de Ciudad Bolívar

En cuanto al estado del arte, para Katharina Vornholt, Patrizia Villottib Beate Muschalla de la revista European Journal Of Work And Organizational Psychology, (2017). Este documento describe el estado de los asuntos de investigación sobre discapacidad y empleo. Por lo tanto, se centra en un grupo particular de personas con discapacidad, enfocándose en la discapacidad mental. Define la discapacidad de acuerdo con la Clasificación Internacional de Funcionamiento, Discapacidad y Salud (ICF) de la Organización Mundial de la Salud, al reconocer que la discapacidad es el resultado de la interacción de la persona y el medio ambiente. Se discuten cuestiones claves, incluidas la complejidad de definir la discapacidad, la situación legal en Europa y América del Norte con respecto a la discapacidad en el trabajo y las barreras y los facilitadores para el empleo. Adicionalmente, se resalta la importancia que tiene este segmento de la población debido a la disminución de la población en edad laboral, especialmente en los países europeos, otorgando un reconocimiento como recurso valioso en la fuerza laboral, es así como, se reconoce la importancia y pertinencia de la investigación sobre discapacidad y empleo. 
En la misma línea, Michael J. Prince (2016) explica que los canadienses con discapacidades han experimentado consistentemente bajos niveles de empleo, así como también barreras en las esferas educativa, económica y social. Enfrentan enormes obstáculos para participar en el mercado laboral, especialmente aquellos con discapacidades severas o bajo nivel educativo. Muchos necesitan adaptaciones y apoyos laborales. En este estudio del IRPP, Michael Prince analiza la situación laboral y el contexto político para adultos en edad laboral con discapacidades mentales o físicas. Él encuentra que un número desproporcionado de ellos están desempleados, incluso si pueden y desean participar en la fuerza laboral. De aquellos que están empleados, muchos trabajan por debajo del salario mínimo y no están protegidos por la legislación laboral. Si bien a lo largo de los años los gobiernos han desarrollado medidas para permitir que los canadienses con discapacidades participen en la fuerza laboral, sus esfuerzos han sido inconsistentes. Los programas federales, provinciales y locales para canadienses con discapacidades son, dice Prince, "un mosaico inconexo de prácticas muy variadas y accesibilidad, accesibilidad y capacidad de respuesta desiguales".

Como respuesta a la dificultad presentada en Canadá, se tienen iniciativas como Bc Partners in Workforce Innovation, el cual tiene como objetivo el incremento del empleo a través de lugares de trabajo inclusivos (2012). Este proyecto buscó demostrar como integrar estrategias de diversidad e inclusión (D\&I) al establecer lugares de trabajo más inclusivos, reclutando talentos centrados en la carrera y mejorar los resultados generales para las personas con discapacidades (Velandia Vargas et al., 2019). A través del puesto de Especialista en Recursos Humanos, el proyecto involucró a los empleadores en la región del Gran Vancouver para asegurar ubicaciones de trabajo para un mínimo de 100 personas con discapacidades / habilidades diversas, con el objetivo de que el 75\% de las personas con discapacidades empleadas sigan empleadas socio empleador o en otro lugar después de seis meses.

Adicionalmente, Un estudio de caso sobre personas finlandesas con discapacidades físicas. (2015) Kasambula Abdul Hakeem, se basa en la revisión de la legislación, las condiciones educativas y sociales que permitan a la población con discapacidad tener una vida independiente, por medio de una vinculación laboral en condiciones óptimas de empleo en la economía formal.

En cuanto a la consulta con fuentes primarias, la población en condición de discapacidad de la localidad Ciudad Bolívar, el 63.2\% presenta discapacidad de carácter cognitivo en un rango de edad de 18 a 35 años, adicional a ello se encontró que en temas de educación, un 45\% no tiene ningún tipo de estudio certificado y un 30\% posee educación básica primaria, lo que nos indica que una de la principales barreras que se presentan a la hora de postularse a una oferta laboral es la falta de competencias certificadas en un $80 \%$, pues es un requisito indispensable para conseguir un empleo en la actualidad. 
Discapacidades de tipo físico o múltiple reflejan barreras como el transporte e infraestructura de las organizaciones, dificultando el recorrido de personas con discapacidad sensorial o impedimentos físicos, sin embargo, aunque presentan barreras de este tipo solo un 21\% de la población cuenta con dichas discapacidades.

La población identifica que al no tener educación certificada no pueden obtener un empleo y al no acceder a este, no logran adquirir experiencia laboral, otro de los más altos requisitos que exigen las empresas según la población. Sin embargo teniendo en cuenta las respuestas obtenidas por la muestra se identificó que dentro de las personas encuestadas se encuentran algunas discapacidades que no logran dar respuesta al instrumento con respecto a su diagnóstico aunque se modificara el lenguaje de la pregunta para que fuese comprendida por la persona, lo que dio como resultado que por sus diagnostico catalogado como severo, se interponía una barrera para lograr obtener una vinculación laboral al momento de presentar entrevistas o que por decisión de sus cuidadores no se ve como una opción el tema laboral ya que a muchos se les dificulta su autocuidado (Camejo Ruiz, 2019).

Frente a la hipótesis planteada sobre si la principal barrera de búsqueda de empleo en las personas en condición de discapacidad de la localidad Ciudad Bolívar es la falta información y de conocimiento sobre ofertas laborales y asesoramiento en temas de inclusión laboral, es verdadera, ya que los resultados arrojaron que la mayoría de la población con un $80 \%$ y $75 \%$ no tiene conocimiento sobre temas laborales, desde agencias de empleo, algún tipo de asesoramiento por parte de alguna entidad, o alguna persona de su círculo social o el conocimiento sobre instituciones que ofrezcan vacantes laborales. La población recibe apoyo en temas sociales, de salud y algunos casos de infraestructura, pero en el tema laboral la localidad no cuenta con vacantes laborales internas y con pocas instituciones que brinden asesoría a dicha población, haciendo el respectivo acompañamiento a la inclusión laboral.

\section{Discusión y Conclusión}

A lo largo del proceso de investigación se descubrió que características de género y edad no eran predominantes para que el individuo con discapacidad no pudieran obtener un empleo laboral, por el contrario se identificó que la falta de información y asesoramiento en procesos laborales se reconoce como una barrera predominante en la búsqueda de empleo para dicha población, por esta razón no acceden a las vacantes laborales, no han trabajado, ni han asistido a entrevistitas; es decir, no ha sido rechazados por las instituciones por que no se postulan a estas. Además, se encontraron dificultades de experiencia y estudios requeridos para acceder a un empleo por lo que optan por no postularse pues le temen al rechazo por no cumplir con los requisitos mínimos que exigen las entidades aun siendo de carácter inclusivo, es por ello que optan por trabajos informarles dentro de la localidad con 
el fin de aportar a sus familias algún ingreso económico, un 65\% de la población se desempeña en actividades informales (García, 2015; Hernández, 2016; Mendoza, 2016).

Las empresas deben reconocer que la población laboralmente activa, posee características diversas y la discapacidad, se debe considerar como una de ellas, lo cual invita a considerar dentro de sus programas de responsabilidad social, el análisis funcional y ocupacional para a identificaciones de actividades y procesos que puedan ser desarrollados por parte de esta población, como aporte a disminuir las brechas de desigualdad y hacer el ejercicio de la inclusión. Por otra parte, se deberá aprender de experiencias exitosas como el caso canadiense en donde los programas gubernamentales, cuentan con especialistas en vinculación laboral que generan vínculos entre la población y los empleadores, generando incentivos de contratación, aportando a la disminución de la desigualdad, la equidad laboral y mitigar los impactos de los riesgos psicosociales. No es suficiente generar espacios culturales y deportivos que les permiten el uso del tiempo libre, sino el logro de una vida productiva e independiente para quienes su condición lo permite(E Norman-Acevedo, 2019).

En el acercamiento a la población, se observó que existe un potencial en talento humano, con la disposición de vincularse a entornos productivos, sin embargo, por el temor al rechazo o porque dichas vacantes no contemplan sus habilidades o no se cuenta con la información suficiente para la preparación al mundo laboral, se abstienen a la postulación.

\section{Referencias bibliográficas}

Camejo Ruiz, A. J. (2019). La emergente ética y responsabilidad social en las relaciones laborales en el siglo XXI (1st ed.). Bogotá: Institución Universitaria Politécnico Grancolombiano.

Daza-Orozco, CE. (2019). Iniciación científica: conceptualización, metodologías y buenas prácticas. Bogotá: Sociedad Colombiana de Investigación e Innovación Formativa - SCOIF. Retrieved from http://www.scoif.com/iniciacioncientifical

De la Cruz Ayala, J. F. Oportunidades de participación laboral en la localidad de bosa para jóvenes entre los 18 y 24 años desplazados por el conflicto armado, una revisión documental (Doctoral dissertation, Universidad del Rosario).

Departamento Administrativo Nacional de Estadísticas, DANE. (15 de marzo de 2019 ). https://www.dane.gov.co/index.php/estadisticas-por-tema/demografia-y-poblacion/discapacidad. Obtenido de https://www.dane.gov.co/index.php/estadisticas-por-tema/demografia-y-poblacion/discapacidad

Farfán Triana, M. H., \& Virgüez Morantes, O. (2018). Análisis de participación en procesos de inclusión en Ciudad Bolívar, de los beneficiarios de la estrategia de inclusión comunitaria del proyecto 1113" Por una ciudad incluyente y sin barreras" (Doctoral dissertation, Corporación Universitaria Minuto de Dios).

Garavito Escobar, D. M. (2014). La inclusión de las personas con discapacidad en el mercado laboral colombiano, una acción conjunta (Doctoral dissertation, Universidad Nacional de Colombia).

García, V. S. (2015). La gestión local como propuesta para la solución de problemas comunes de urbanismo en América Latina. Panorama, 9(16), 92-103. https://doi.org/10.15765/pnrm.vgi16.633

Hernández Sampieri, R., Fernández Collado, C., \& Baptista Lucio, P. (2010). Metodología de la investigación.

Hernández, Y. M. (2016). La Unión Patriótica: memorias para la paz y la democracia. Panorama, 10(18), 27-38. https://doi.org/10.15765/pnrm.v10i18.822

Kasambula Abdul Hakeem, (2015). Tesis Personas con discapacidad física y su vida de trabajo, Universidad de Ciencias Aplicadas, Programa de Licenciatura en Servicios Sociales.

Katharina Vornholt, Patrizia Villotti, Beate Muschalla, Jana Bauer, Adrienne Colella, Fred Zijlstra, Gemma Van Ruitenbeek, Sjir Uitdewilligen \& Marc Corbi ère (2017): Discapacidad y empleo: Descripción general y detalles destacados, European Journal of Work and Organizational Psychology, DOI: 10.1080 / 1359432X.2017.13875 
Luna, González Claudia María, Montes, Ballestas Triana Marcela (2017). Proyecto de grado: Inclusión laboral de personas en condición de discapacidad física, En el sector servicios; call centers. Universidad Externado de Colombia.

Martínez-Rozo, A. M., Uribe-Rodríguez, A. F., \& Velázquez-González, H. J. (2015). La discapacidad y su estado actual en la legislación colombiana. Duazary, 12(1), 49 - 58. https://doi.org/10.21676/2389783X.1398.

Mendoza, J. A. G. (2016). Empresa privada: principal socio en el posconflicto y la construcción de la paz. Panorama, 10(18), 8492. https://doi.org/10.15765/pnrm.v10i18.823

Navarrete, E. L., Padrón Innamorato, M., y Silva Arias, A. C. (2013). La inserción laboral de los jóvenes y las políticas de empleo en Colombia, México y Uruguay (2012). Población y trabajo en América Latina: abordajes teórico-metodológicos y tendencias empíricas recientes. Río de Janeiro, Brasil.

Norman-Acevedo, E. (2018). Rompiendo Barreras: 10 Años de la Educación Virtual en el Politécnico Grancolombiano. Bogotá: Institución Universitaria Politécnico Grancolombiano. Retrieved from http://alejandria.poligran.edu.co/handle/10823/1146

Norman-Acevedo, E. (2019). Consumer cultural studies. Bogotá: Institución Universitaria Politécnico Grancolombiano.

Prince, M.J. 2016. Empleo inclusivo para canadienses con discapacidades: hacia un nuevo marco y agenda de políticas, estudio IRPP 60. Montreal: Instituto de Investigación sobre Políticas Públicas

Velandia Vargas, G. K., Malangón Torres, M. A., Ramos Duarte, N., Quiroz Rubiano, M. M., Rodríguez Rubio, A., \& Celemín Pabón, Y. A. (2019). Experiencias de innovación educativa - Tomo 2 (1st ed.; L. M. Trujillo-Flórez, ed.). Bogotá: Politécnico Grancolombiano.

Velandia Vargas, G. K., Malangón Torres, M. A., Ramos Duarte, N., Quiroz Rubiano, M. M., Rodríguez Rubio, A., \& Celemín Pabón, Y. A. (2019). Experiencias de innovación educativa - Tomo 2 (1st ed.; L. M. Trujillo-Flórez, ed.). Bogotá: Politécnico Grancolombiano. 